\title{
Statistical Shape Modeling using Morphological Representations
}

\author{
Santiago Velasco-Forero and Jesús Angulo \\ Centre de Morphologie Mathématique, Mathématiques et Systèmes, MINES ParisTech \\ E-mail: santiago.velasco/jesus.angulo@mines-paristech.fr
}

\begin{abstract}
The aim of this paper is to propose tools for statistical analysis of shape families using morphological operators. Given a series of shape families (or shape categories), the approach consists in empirically computing shape statistics (i.e., mean shape and variance of shape) and then to use simple algorithms for random shape generation, for empirical shape confidence boundaries computation and for shape classification using Bayes rules. The main required ingredients for the present methods are well known in image processing, such as watershed on distance functions or log-polar transformation. Performance of classification is presented in a well-known shape database.
\end{abstract}

\section{Introduction}

Object recognition based on shape information is a classic problem in image processing. That motivates the development of tools for statistical analysis of shapes in $\mathbb{R}^{2}$. In [5] is described a geometric technique to parametrize curves based on their arc lengths and their angle function to represent and analyze shapes. Techniques for clustering learning in planar shapes are described in [9]. Shape classification problem is referred as "classify a given shape into one of the predefined classes", it can be solved using a shape-probability per class. Usually the first step towards the design of a shape classifier is a feature extraction followed by a shape matching stage. Curvature, chain codes, Fourier descriptors, tangent space representation [6] and beam angle statistics (BAS) [2] are examples of contourbased descriptors. Dynamic programming (DP)-based shape matching is applied successfully in [8] for shape matching. Hidden Markov models (HMMs) are also being explored as one of the possible shape modeling and classification frameworks [11].

This paper presents an approach to analyze a given family of shapes based on the computation of mean shape and associated variance of shape, obtained by means of a mathematical morphology algorithm. Starting for the empirically obtained mean and variance, it is introduced a novel methodology of shape classification, using a training stage producing an associated probability map per class. Additionally, random shape generation and empirical confidence shape boundaries computation are also investigated. All the examples of the paper are obtained with shape families of the MPEG7 database. Additional examples of the different algorithms using the complete database can be found in the website ${ }^{1}$.

\section{Statistical Shape Modelling}

\subsection{Mean shape and variance of shape using mathematical morphology}

Let $\mathcal{X}_{j}=\left\{X_{1}^{j}, X_{2}^{j}, \ldots, X_{N_{j}}^{j}\right\}$ be a family (or collection) of $N_{j}$ shapes for the class $j$, for $j=1, \ldots, J$ where $X_{i}^{j} \in P(E)$ represents the set (or binary image) of the shape $i$ in the family of shapes labeled by $j$, and the support space $E$ is a nonempty set. Typically for the digital 2D images $E \subseteq Z^{2}$. The set $X_{i}^{j}$ is a compact set (and typically a closed simply connected set). The family $\mathcal{X}_{j}$ can be considered as a random variable of shape, where $X_{i}^{j}$ represents a realization of this random variable. We make the assumption that each class of shapes, i.e. the set of shapes that can be identified with a common concept or object, e.g. fish shapes, are they are centered, oriented and scaled.

Let us start by the classical definition of the mean of $N$ samples: $\mu$ is the value such that $f(c)=\sum_{i}^{N}\left(x_{i}-\right.$ $c)^{2}$ is minimal. In the extension to a shape family $\mathcal{X}_{j}$, we start by constructing the sum of distance functions to the frontier sets $\Upsilon\left(\mathcal{X}_{j}\right)$ using the squared Euclidean distance, or an appropriate squared discrete distance [3] ( $\Delta_{c}^{2}$ denote outer distance and $\Delta^{2}$ inner distance). Thus, the mean shape $\mu_{\mathcal{X}_{j}} \in P(E)$ is the compact set associ-

\footnotetext{
${ }^{1}$ http://cmm.ensmp.fr/ velasco/Shape
} 


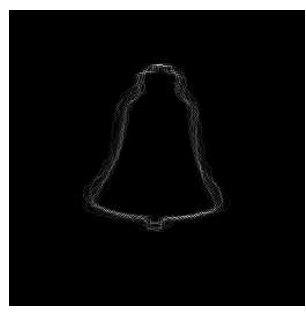

(a) $\mathcal{X}_{j}$

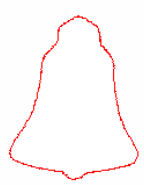

(c) $\Upsilon\left(\mu\left(\mathcal{X}_{j}\right)\right)$

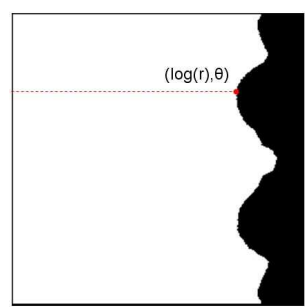

(e) $\mu_{\mathcal{X}_{j}}^{p}$

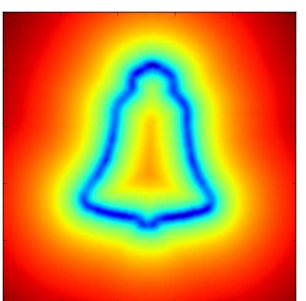

(b) $\Delta^{2} \mathcal{X}_{j}$

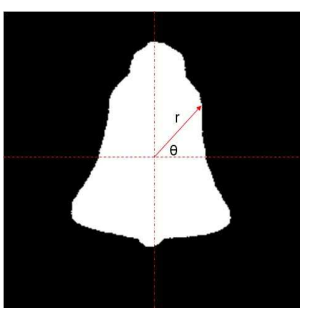

(d) $\mu_{\mathcal{X}_{j}}$

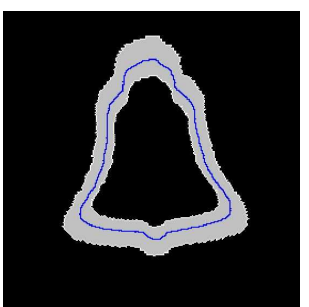

(f) $95 \%$-ECB for $\mu\left(\mathcal{X}_{j}\right)$

\section{Figure 1. Analysis of "bell" shapes using} the proposed approach.

ated to of locally minimal contour of obtained from the functional:

$$
\Delta^{2} \mathcal{X}_{j}=\sum_{i}^{N}\left[\Delta_{c}^{2} X_{i}^{j}+\Delta^{2} X_{i}^{j}\right]
$$

The contour, or frontier, of the mean shape $\mu_{\mathcal{X}_{j}}$ will be denoted by $\Upsilon\left(\mu_{\mathcal{X}_{j}}\right)$. As it was introduced in [1], this optimal contour can be easily obtained by computing the watershed line of the inverse (or complement) of $\Delta^{2} \mathcal{X}_{j}$. An example of this analysis is given in Fig. 1. Additionally, using also the generalization of the classical statistical notion, the variance of a shape family is defined for each point in the mean contour as the function $\sigma_{\mathcal{X}_{j}}: \Upsilon\left(\mu_{\mathcal{X}_{j}}\right) \rightarrow \mathbb{R}^{+}$, given by [7]:

$$
\sigma_{\mathcal{X}_{j}}=\frac{\Delta^{2} \mathcal{X}_{j}}{N_{j}}
$$

It is important to remark that the variance of shape is a set of nonnegative values associated to each point in $\Upsilon\left(\mu_{\mathcal{X}_{j}}\right)$.

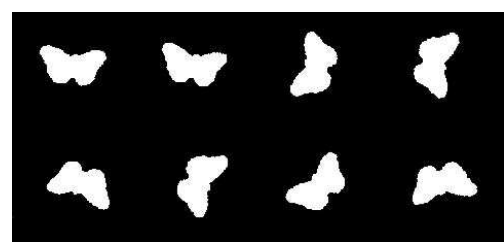

(a) Random Shapes

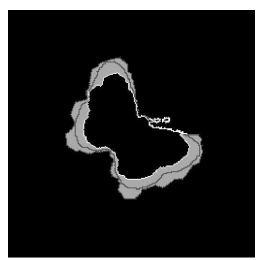

(b) ECB for $\Upsilon\left(\mu_{\mathcal{X}_{j}}\right)$ and $\alpha=0.95$

\section{Figure 2. Random shapes and empirical confidence boundaries for $\Upsilon\left(\mu_{\mathcal{X}_{j}}\right)$ in the "Butterfly" shapes family}

\subsection{Random Shapes Generation}

A straight statistical application of the representation $\left(\mu_{\mathcal{X}_{j}}, \sigma_{\mathcal{X}_{j}}\right)$ is the generation of random shapes. Our proposal is to generate a random perturbation of $\mu_{\mathcal{X}_{j}}$ in its log-polar representation [1], and these stochastic perturbations must follows the empirical variance $\sigma_{\mathcal{X}_{j}}$. More precisely, the approach is detailed in algorithm 1. Fig.1(e) $\mu_{\mathcal{X}_{j}}^{p}$ gives the log-polar coordinate representation (superscript ${ }^{p}$ will denote log-polar space) of the mean shape depicted in Fig.1(d). An example of random shape generation according to our algorithm, for a family of butterflies, is presented in Fig. 2(a). More examples can be found in the website ${ }^{1}$.

$$
\begin{aligned}
& \text { Input : Family of shape } \mathcal{X}_{j} \\
& \text { output: Random shape } R\left(\mathcal{X}_{j}\right)
\end{aligned}
$$

Calculate $\mu_{\mathcal{X}_{j}}$ and $\sigma_{\mathcal{X}_{j}}$;

Transform to $\log$-polar $\mu_{\mathcal{X}_{j}}^{p}$ and $\sigma_{\mathcal{X}_{j}}^{p}$;

$u_{1}=U(-1,1), u_{2}=U(0,2 \pi)$;

for $i \leftarrow 1$ to $2 \pi$ do

| $R^{p}\left(\theta_{i}, \bmod \left(:+u_{2}, 2 \pi\right)\right)=\mu_{\mathcal{X}_{j}}^{p}\left(\theta_{i},:\right)+u_{1} \sigma_{\mathcal{X}_{j}}^{p}\left(\theta_{i},:\right) ;$

end

$R=$ polar inverse transformation of $R^{p}$;

Algorithm 1: Random Generation of Shapes

\subsection{Empirical Confidence Boundaries for shape mean}

Under the assumption of independence gaussian distribution in each direction of the log-polar transformation, i.e., $\mathcal{N}\left(0, \sigma_{\mathcal{X}_{j}}^{p}\left(\theta_{i}\right)\right)$, we can define the notion of Empirical Confidence Boundaries (ECB) of shape. The idea is to extract a confidence boundary around $\mu_{\mathcal{X}_{j}}$ using the percentiles associated to a gaussian distribution. Technical description of our approach is given in algorithm 2 , where $\Delta$ denotes the symmetric set difference and $\Phi^{-1}$ represents the inverse of the standardized 
gaussian cumulative distribution function. Examples of ECB of shape are showed in Figs. 2(b) and 1(f).

Input : Family of shape $\mathcal{X}_{j}$, confidence parameter $\alpha$ output: Boundary $C B_{\alpha}$

Calculate $\mu_{\mathcal{X}_{j}}$ and $\sigma_{\mathcal{X}_{j}}$;

Transform to $\log$-polar $\mu_{\mathcal{X}_{j}}^{p}$ and $\sigma_{\mathcal{X}_{j}}^{p}$;

$k_{1}=\Phi_{\left(1-\alpha_{2}\right)}^{-1}$;

$k_{2}=\Phi_{\left(\alpha_{2}\right)}^{-1}$;

for $i \leftarrow 1$ to $2 \pi$ do

$$
\begin{aligned}
& S_{1}^{p}\left(\theta_{i}\right)=\mu_{\mathcal{X}_{j}}^{p}\left(\theta_{i}\right)+k_{1} \sigma_{\mathcal{X}_{j}}^{p}\left(\theta_{i}\right) \\
& S_{2}^{p}\left(\theta_{i}\right)=\mu_{\mathcal{X}_{j}}^{p}\left(\theta_{i}\right)+k_{2} \sigma_{\mathcal{X}_{j}}^{p}\left(\theta_{i}\right)
\end{aligned}
$$

end

$C B_{\alpha}=S_{1} \Delta S_{2}$

Algorithm 2: Empirical confidence boundary of a shapes family

\subsection{Classification in families of shapes}

Starting again from the pair $\left(\mu_{\mathcal{X}_{j}}, \sigma_{\mathcal{X}_{j}}\right)$, the goal now is to define a probability associated to the family of shapes $\mathcal{X}_{j}$. Firstly, we introduce a measure for a point $\overrightarrow{v_{i}} \in \mathbb{R}^{2}$ w.r.t. the shape family $\mathcal{X}_{j}$ as follows:

$$
\Psi_{j}\left(\vec{v}_{i}\right)=\max _{k} \mathcal{N}\left(\Upsilon\left(\mu_{\mathcal{X}_{j}}\right)(k), \Sigma_{\mathcal{X}_{j}}(k)\right)\left(\overrightarrow{v_{i}}\right)
$$

where the bivariate gaussian distribution is centered at each point $k$ which belongs to the mean contour $\Upsilon\left(\mu_{\mathcal{X}_{j}}\right)(k)$ and has an uncorrelated covariance matrix $\Sigma_{k}=\mathbf{I}_{(2 \times 2)} \sigma_{\mathcal{X}_{j}}(k)$, where $\sigma_{\mathcal{X}_{j}}(k)$ is the variance value at point $\Upsilon\left(\mu_{\mathcal{X}_{j}}\right)(k)$. The gaussian distribution in expression (3) can be clearly substituted for another elliptically contoured distributions or even an asymmetric distribution to penalize any direction. Fig. 3(a) shows an illustration of the assumption of bivariate gaussian distribution at each value of $\Upsilon\left(\mu_{\mathcal{X}_{j}}\right)$. Secondly, given a set of shapes $\mathcal{X}_{j}$, their associated probability map (APM) is defined as

$$
A P M_{j}=\Psi_{j}\left(\overrightarrow{v_{i}}\right), \forall \overrightarrow{v_{i}} \in \mathbb{R}^{2}
$$

Under bivariate gaussian assumption, the expression (4) can be calculated for a given point $\vec{v}$ as

$$
\begin{aligned}
A P M_{j} & =\max _{k} \int_{0}^{2 \pi} \int_{0}^{c_{k}} r \exp ^{\frac{-r^{2}}{2 \sigma^{2}}} d r d \theta \\
& =1-\min _{k}\left\{\exp ^{\frac{-c_{k}^{2}}{2 \sigma_{k}^{2}}}\right\}
\end{aligned}
$$

where $c_{k}^{2}$ is the distance between $\vec{v}$ and each point $k \in$ $\Upsilon\left(\mu_{\mathcal{X}_{j}}\right)$. Fig. 3(b) shows the $A P M_{j}$ for the class of bells. Other examples can be consulted in the website ${ }^{1}$.

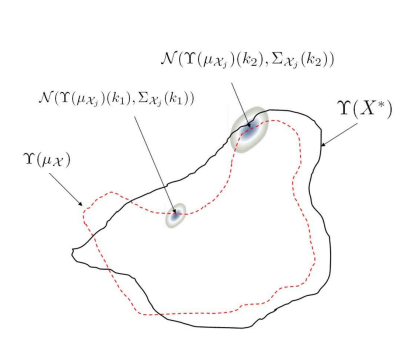

(a) Bivariate gaussian assumption in each value of $\Upsilon\left(\mu_{\mathcal{X}}\right)$

\section{Figure 3. Shape family, mean and variance contours using proposed approach.}

The next step is to use $A P M_{j}$ in order to obtain an analytical expression to calculate the empirical conditional probability per class $j$ for a new shape $X^{*} \in$ $P(E)$, denoted $P\left[J=j / X^{*}\right]$, knowing the empirical mean $\mu_{\mathcal{X}_{j}}$ and variance $\sigma_{\mathcal{X}_{j}}$. Intuitively, a shape $X^{*}$ going through zones of the space with highest probability $A P M_{j}$ has to accumulate more likely in it. On the other hand, if $\int \Upsilon\left(X^{*}\right) \ll \int \Upsilon\left(\mu_{\mathcal{X}_{j}}\right)$, its probability has to reduce, because we assume that all the shapes are oriented and rescaled. Therefore, taking into account these ideas, the a posteriori probability is given by

$$
P\left[J=j / X^{*}\right]=\frac{\int_{A P M_{j}} X^{*}}{\max \left(\int X^{*}, \int \Upsilon\left(\mu_{\mathcal{X}_{j}}\right)\right)}
$$

where $\int$ denote the integral over an APM with constant value 1 for every point. Note that the denominator is comparing lengths between the frontier of the mean shape in the class $j$ and the frontier of the new shape to predict.

Consequently, we can use (5) in a Bayes decision rule to design the following statistical classifier $\mathbf{g}(\cdot)$ :

$$
\mathbf{g}\left(X^{*}\right)=\underset{j}{\operatorname{argmax}}\left(\pi_{j} P\left[J=j / X^{*}\right]\right)
$$

where $\pi_{j}$ is the a priori probability and it represents the likelihood for the appearance of the class $j$ in the global data set.

\section{Experiments}

The well-known MPEG-7 test database consists of 70 types of objects, each family having 20 different shapes. The database is challenging due to the presence of examples that are visually dissimilar from other members of their class, and examples that are highly 
Table 1. Performance comparison of shape classification for MPEG-7 database

\begin{tabular}{|c|c|}
\hline Method & Accuracy (\%) \\
\hline Robust Symbols Representation [4] & 98.57 \\
RACER [10] & 96.80 \\
Our method & 96.83 \\
\hline
\end{tabular}

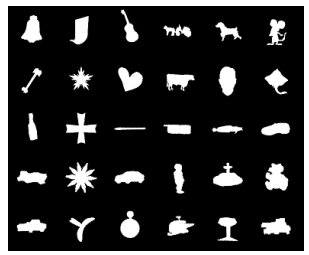

Figure 4. A representation for each class considered in experimental results

similar to members of other classes. Our experiments involve a subset of the available shapes. The subset consists of 600 images: 30 shape categories, 20 images per category. Shapes have been centered using center of gravity, oriented using the principal axis and finally, their size have been normalized using the minimal bounding circle in log-polar representation. We consider uninformative a priori probabilities in expression (6). Fig. 4 shows an example of one shape for all the classes considered. To evaluate the accuracy of classification using the proposed approach, we have used cross validation Leave-one-Out strategy. Results of $A S M$ computation and shape classification are presented in Fig. 5(a) and Table 1 (which includes the comparison with other published works). Average confusion matrix is given in Fig. 5(b).

\section{Conclusion and Perspectives}

A new approach for the statistical analysis of 2-D shapes has been presented. We have shown how our methodology can be used for shape classification using a quite simple probabilistic formulation, by computing an associated probability map per class and using a simple Bayes classifier as classification rule. The results indicate that the classification performance is comparable to those reported in the literature using others approaches. Moreover, an algorithm of shape random generation has been also presented, and an empirical approach to produce confidence boundaries has been formulated. Finally, we notice that our approach does not require feature extraction and it is formulated in the nat-

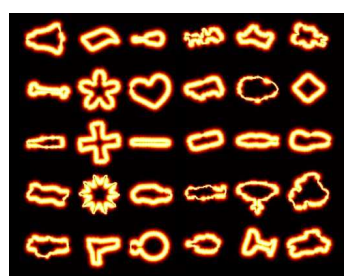

(a) ASM per class

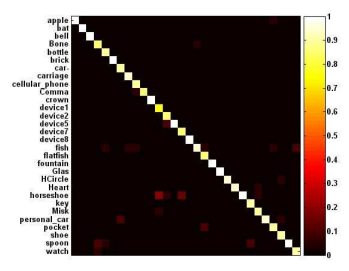

(b) Confusion Matrix

\section{Figure 5. Associated probability map and confusion matrix for MPEG-7}

ural form of a shape space. Future work will deal with a more formal mathematical framework for the notion of "measure" of this shape space. Random sampling based in gaussian processes, considering spatial correlation, will be considered in future research.

\section{References}

[1] J. Angulo and F. Meyer. Morphological exploration of shape spaces. In ISMM '09, pages 226-237. SpringerVerlag, 2009.

[2] N. Arica and F. T. Y. Vural. Bas: a perceptual shape descriptor based on the beam angle statistics. Pattern Recogn. Lett., 24(9-10):1627-1639, 2003.

[3] G. Borgefors. In Handbook of Pattern Recognition and Computer Vision, 3rd Edition, pages 157-176.

[4] M. R. Daliri and V. Torre. Robust symbolic representation for shape recognition and retrieval. Pattern Recogn., 41(5):1782-1798, 2008.

[5] E. Klassen, A. Srivastava, W. Mio, and S. H. Joshi. Analysis of planar shapes using geodesic paths on shape spaces. IEEE Trans. Patt. Anal., 26(3):372-383, 2004.

[6] L. J. Latecki and R. Lakamper. Shape similarity measure based on correspondence of visual parts. IEEE Trans. Pattern Anal. M.I., 22:1185-1190, 2000.

[7] M. A. Luengo-Oroz and J. Angulo. Cyclic mathematical morphology in polar-logarithmic representation. IEEE Trans. Img. Proc., 18(5):1090-1096, 2009.

[8] E. G. M. Petrakis, A. Diplaros, and E. Milios. Matching and retrieval of distorted and occluded shapes using dynamic programming. IEEE Trans. Pattern Anal. M.I., 24(11):1501-1516, 2002.

[9] A. Srivastava, S. H. Joshi, W. Mio, and X. Liu. Statistical shape analysis: Clustering, learning, and testing. IEEE Trans. Pattern Anal. M.I., 27(4):590-602, 2005.

[10] B. J. Super. Improving object recognition accuracy and speed through nonuniform sampling. In D. P. Casasent, E. L. Hall, \& J. Röning, editor, SPIE, volume 5267 of (SPIE) Conference Series, pages 228-239, Oct. 2003.

[11] N. Thakoor, J. Gao, and S. Jung. Hidden markov modelbased weighted likelihood discriminant for 2-d shape classification. IEEE Trans. Img. Proces., 16(11):27072719, 2007. 